\title{
CESE Report \\ A Patient with Cavernous Sinus Dural Arteriovenous Fistula Complicating Klippel-Trenaunay Syndrome
}

Etsuko Owashi, ${ }^{1}$ Takayuki Kato, ${ }^{1}$ Jouji Kokuzawa, ${ }^{2}$ Hirofumi Matsubara, ${ }^{1}$ Tatsuki Aki, ${ }^{1}$ Shin-ichi Shirakami, ${ }^{1}$ and Shu Imai ${ }^{1}$

Objective: We report a case of cavernous sinus dural arteriovenous fistula (CSdAVF) associated with Klippel-Trenaunay syndrome (KTS).

Case Presentation: A 58-year-old woman was diagnosed with KTS with port-wine stain, overgrowth of tissues and bones, and venous malformation on the left upper limb. She was admitted to our hospital with the primary complain of ptosis and diplopia due to left oculomotor palsy, and her cerebral angiography revealed CSdAVF with retrograde leptomeningeal venous drainage. The shunt point was located at the posteromedial part of the left cavernous sinus (CS) on the angiogram. An enlarged subclavian vein and giant varix was detected in the left upper limb, and abnormality of the coagulationfibrinolysis system caused by localized intravascular coagulation was confirmed. We performed transvenous coil embolization, and the symptoms improved after a few weeks with no new neurological deficits. However, the activation of coagulation-fibrinolysis system continued even after the surgery.

Conclusion: The formation of dAVF occurrence in this case is unclear. If genetic abnormalities that cause angiogenesis are involved in KTS, follow-up is important in the future.

Keywords \ Klippel-Trenaunay syndrome, dural arteriovenous fistula, transvenous embolization, venous malformation, coagulopathy

\section{Introduction}

Dural arteriovenous fistula (dAVF) is a disease with acquired shunt formation in the dura, and the annual incidence is reported to be $0.16 / 100000$ people overseas ${ }^{1)}$ and $0.29 / 100000$ people in Japan. ${ }^{2}$ Inducers of dAVF include head injury and infection, past medical history of craniotomy, venous thrombosis due to abnormal coagulation, and venous hypertension, but the cause is unclear in many cases.

${ }^{1}$ Department of Neurosurgery, Daiyukai General Hospital, Ichinomiya, Aichi, Japan

${ }^{2}$ Department of Neurosurgery, Asahi University Hospital, Gifu, Gifu, Japan

Received: December 19, 2018; Accepted: July 24, 2019

Corresponding author: Etsuko Owashi. Department of Neurosurgery, Daiyukai General Hospital, 1-9-9 Sakura, Ichinomiya, Aichi 491-8551, Japan

Email: neuro.daiyukai+O.E@gmail.com

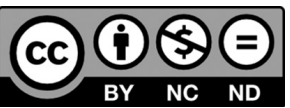

This work is licensed under a Creative Commons Attribution-NonCommercialNoDerivatives International License.

(C)2019 The Japanese Society for Neuroendovascular Therapy
In the neurosurgery field, hemangioma on the body surface and in soft tissue and vascular malformation are rarely encountered. Multiple or very large vascular malformations are resistant to various treatments and life-long treatment is necessary due to pain, ulcer formation, abnormal growth of the affected limb, and problems with the appearance. We encountered a patient with dAVF complicating Klippel-Trenaunay syndrome (KTS), which is classified as vascular malformation-related syndrome.

\section{Case Presentation}

Patient: 58-year-old female patient.

Chief complaints: Left ptosis and diplopia.

Past medical history: Port-wine stains in the left upper limb and left precordium have been pointed out since birth, a varix was noted in the left upper limb as a juvenile, and the patient was diagnosed with KTS. In addition, the left pointing finger was amputated due to persistent hemorrhage, although the details were unclear. The patient regularly visited the hospital for venous hemorrhage from peripheral ulcer in the left upper limb and received sclerotherapy from 1 year earlier, but she had no previous medical history 


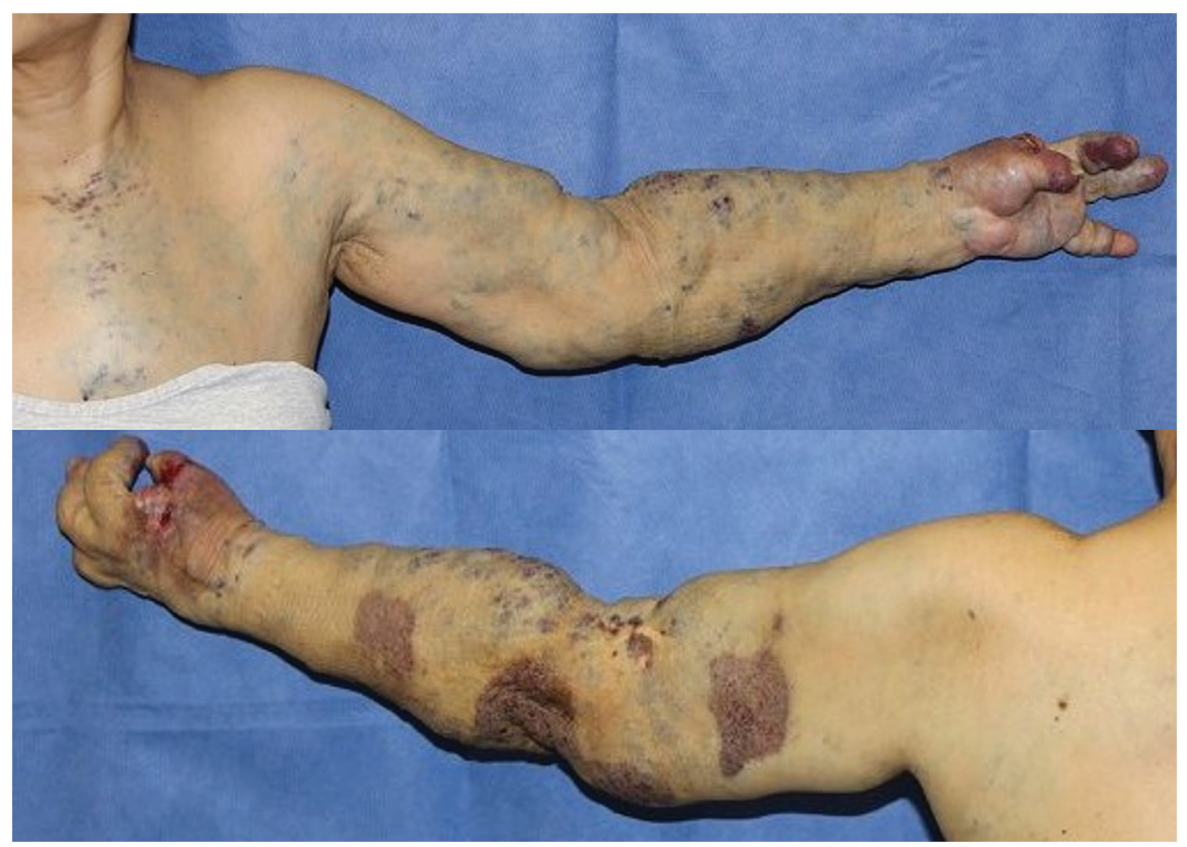

Fig. 1 Pigmented nevus and varix were noted on the left upper limb over the left precordium. The upper limb including the fingers were enlarged.
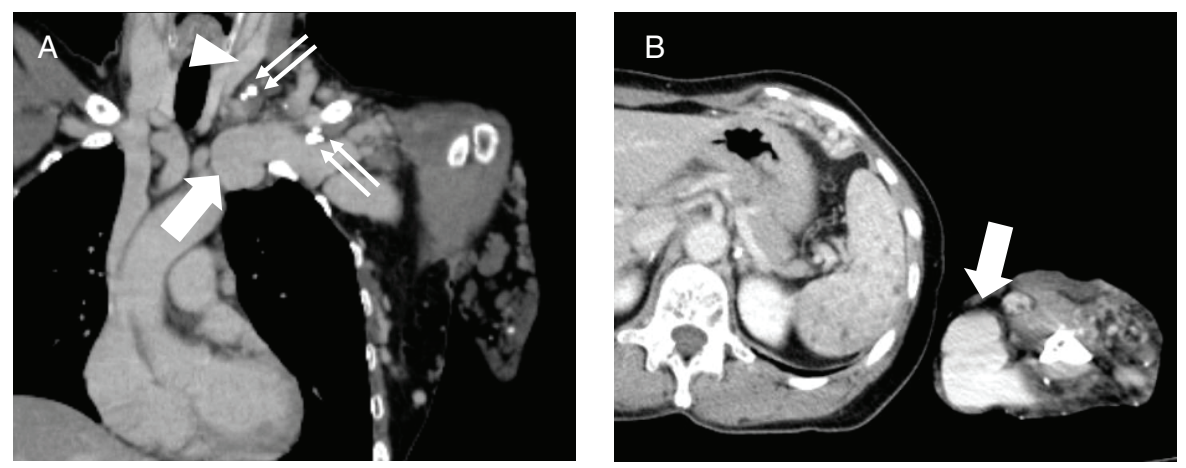

Fig. 2 Delayed phase of contrast-enhanced CT. (A) Coronal view. The subclavian vein was markedly dilated, but the left internal jugular vein was not dilated. Phleboliths were present in the subclavian vein. (B) Axial view. A megavein was present in the medial upper arm. arrow: subclavian vein, arrowhead: internal jugular vein, double arrow: phleboliths.

of head injury and malignant disease or treatment with an oral antithrombotic or anticoagulant.

History of present illness: The disease suddenly manifested as left ptosis and diplopia. The patient visited a physician and was referred to our hospital, suspecting an intracranial lesion. On the first examination, consciousness was clear, left conjunctival hyperemia, left oculomotor paresis, and left pulsatile tinnitus were observed, the affected left upper limb was entirely swollen, and a dilated varix was present (Fig. 1). On close examination, Borden type II cavernous sinus (CS) dAVF was observed, in which blood flows from the left middle meningeal artery, accessory meningeal artery, artery of foramen rotundum, and ascending pharyngeal artery into the medial posterior part of the left CS and flowed out into the left superior ophthalmic vein (SOV), superficial middle cerebral vein (SMCV), inferior petrosal sinus (IPS), and pterygoid plexus, and it was accompanied by cortical venous reflux. In addition, the shunt also flowed into the lateral CS. In the delayed phase of contrast-enhanced CT of the trunk, a giant varix was present in the left upper limb and vascular dilatation expanded to the left subclavian vein, but the left internal jugular vein was not dilated (Fig. 2). Many phleboliths were present in the left upper limb, but no filling defect suggesting thrombus was present in the varix (Fig. 3). On 


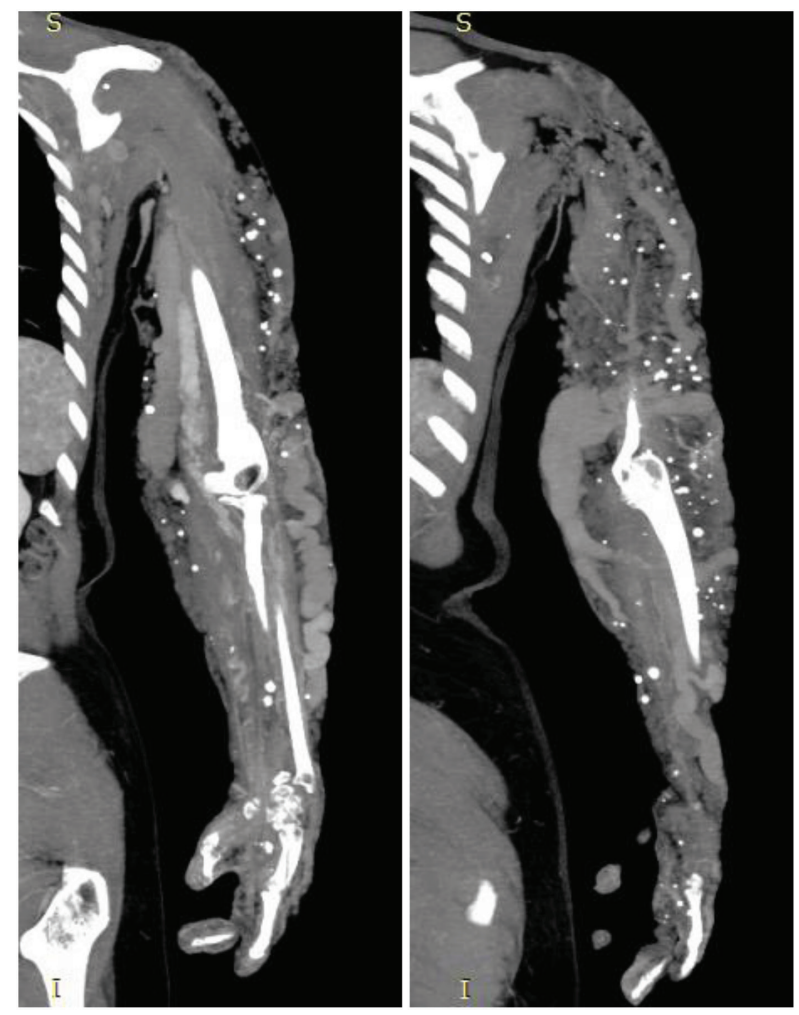

Fig. 3 Contrast-enhanced CT MIP. A varix and many phleboliths were present in the upper arm over the forearm. MIP: maximum intensity projection

blood testing, the platelet count was $10.6 \times 10^{4} / \mu \mathrm{L}$; fibrinogen, $104.6 \mathrm{mg} / \mathrm{dL}$; fibrin degradation product (FDP), $39.1 \mu \mathrm{g} / \mathrm{mL}$; D-dimer, $20.5 \mu \mathrm{g} / \mathrm{mL}$; showing abnormality of the coagulation-fibrinolysis system (Table 1). For treatment, transvenous coil embolization (TVE) with heparinization only during treatment without pre- or postoperative antithrombotic therapy was planned.

Treatment course: The right common femoral artery was punctured and a catheter for diagnosis was placed in the left external carotid artery. The right femoral vein was punctured and 6Fr Launcher (Medtronic, Minneapolis, MN, USA) was placed in the left internal jugular vein and advanced to the CS through the IPS using 4Fr BHW (Katecs, Aichi, Japan) and Radifocus Guidewire 0.035 (Terumo, Tokyo, Japan). Using this as an index, SL-10 (Stryker, Kalamazoo, MI, USA) was guided to the CS using GT wire 0.012 angle (Terumo). In the CS, SL-10 was advanced to the anterior compartment, and first, the SMCV was embolized with Target 360 (Stryker) and Axium helix (Medtronic). Then, the SOV was embolized with Deltamaxx (Codman Neuro) and Target 360, followed by embolization of the posteromedial compartment of the CS, in which the shunt point was present, with Axium 3D, Target
360, and Galaxy complex (Codman Neuro). When angiography was performed at this time-point, the SOV and cortical reflux disappeared and the shunt to the CS was also lost so that the procedure was completed (Fig. 4). Pulsatile tinnitus resolved and ptosis improved immediately after treatment, but pulsatile tinnitus appeared 10 days after treatment. On catheter examination, reflux to the SOV and $\mathrm{SMCV}$ in dominance with the former was noted and additional treatment was planned.

At 2 weeks after the first treatment, SL-10 was guided to the CS through the IPS after right femoral vein puncture as described above. Reflux to the SOV had resolved but reflux to the SMCV had aggravated due to re-canalization of the region embolized in the first treatment. The region of the SMCV embolized in the first treatment over the proximal region was embolized, followed by additional embolization of the posteromedial compartment of the CS, and the shunt disappeared (Fig. 5). Pulsatile tinnitus disappeared immediately after treatment, and oculomotor paralysis completely resolved 3 weeks after the second treatment. No recurrence has occurred for 3 years thereafter.

\section{Discussion}

KTS is a syndrome of congenital abnormal vasculogenesis developing port-wine stains in the four limbs, varix, and bone soft tissue overgrowth as the three main symptoms, and it develops in the unilateral lower limb in $75 \%$ of cases. In the classification established by the International Society for the Study of Vascular Anomalies (ISSVA) classification, ${ }^{3)}$ simple vascular malformation is classified into capillary blood vessel malformation, lymph vessel malformation, venous malformation, arteriovenous malformation (AVM), and arteriovenous fistula, and in the mixed type, several vascular malformations are present. KTS is complicated by capillary blood vessel malformation, lymph vessel malformation, and venous malformation in many cases and enlargement of bone soft tissue accompanies as a concomitant symptom so that it is classified as a related syndrome in the ISSVA classification. It is considered that KTS, in which the flow rate is low, should be distinguished from Parkes Weber syndrome (PWS) complicated with high-flow-rate arteriovenous fistula, hyperplasia in the affected limb, and heart failure in severe cases. However, both syndromes were collectively reported as Klippel-Trenaunay Weber Syndrome (KTWS) in many previous studies.

KTS-associated complication by vascular lesions in the central nervous system has been reported. In all, 12 cases 
Table 1 Time-course changes in the platelet count and values of the coagulation-fibrinolysis system in the treatment course

\begin{tabular}{|c|c|c|c|c|c|c|c|c|c|c|}
\hline & pre $12 \mathrm{M}$ & pre 7D & 1D & $3 D$ & $7 \mathrm{D}$ & $13 \mathrm{D}$ & $6 \mathrm{M}$ & $12 \mathrm{M}$ & $24 \mathrm{M}$ & $36 \mathrm{M}$ \\
\hline Platelet $\left(14-38 \times 10^{4} / \mu \mathrm{L}\right)$ & 12.9 & 10.6 & 11.1 & 10.1 & 12.7 & 11.5 & 10.8 & 11.0 & 11.4 & 10.2 \\
\hline Fibrinogen $(150-400 \mathrm{mg} / \mathrm{dL})$ & & 104.6 & 108.8 & 56.1 & 73.5 & 169.0 & 165.4 & 178.0 & 149.1 & 167.4 \\
\hline $\operatorname{FDP}(<10 \mu \mathrm{g} / \mathrm{mL})$ & 38.4 & 39.1 & 230.0 & 97.2 & 91.9 & 81.0 & 55.0 & 37.4 & 80.6 & 72.8 \\
\hline D-dimer (<5 ng/mL) & 23.6 & 20.5 & 91.8 & 45.2 & 40.3 & 37.0 & 29.1 & 20.4 & 33.5 & 37.7 \\
\hline TAT (<3 ng/mL) & & & & & 70.8 & & & & & 273.0 \\
\hline $\mathrm{PIC}(<0.8 \mu \mathrm{g} / \mathrm{mL})$ & & & & & 9.0 & & & & & 4.6 \\
\hline
\end{tabular}

The treatment course is presented as the number of days from the first treatment. The platelet count was low and the FDP and D-dimer levels were high throughout the course. D: date; FDP: fibrin degradation product; M: month; TAT: Thrombin-anti thrombin complex; PIC: Plasmin-inhibitor plasmin complex

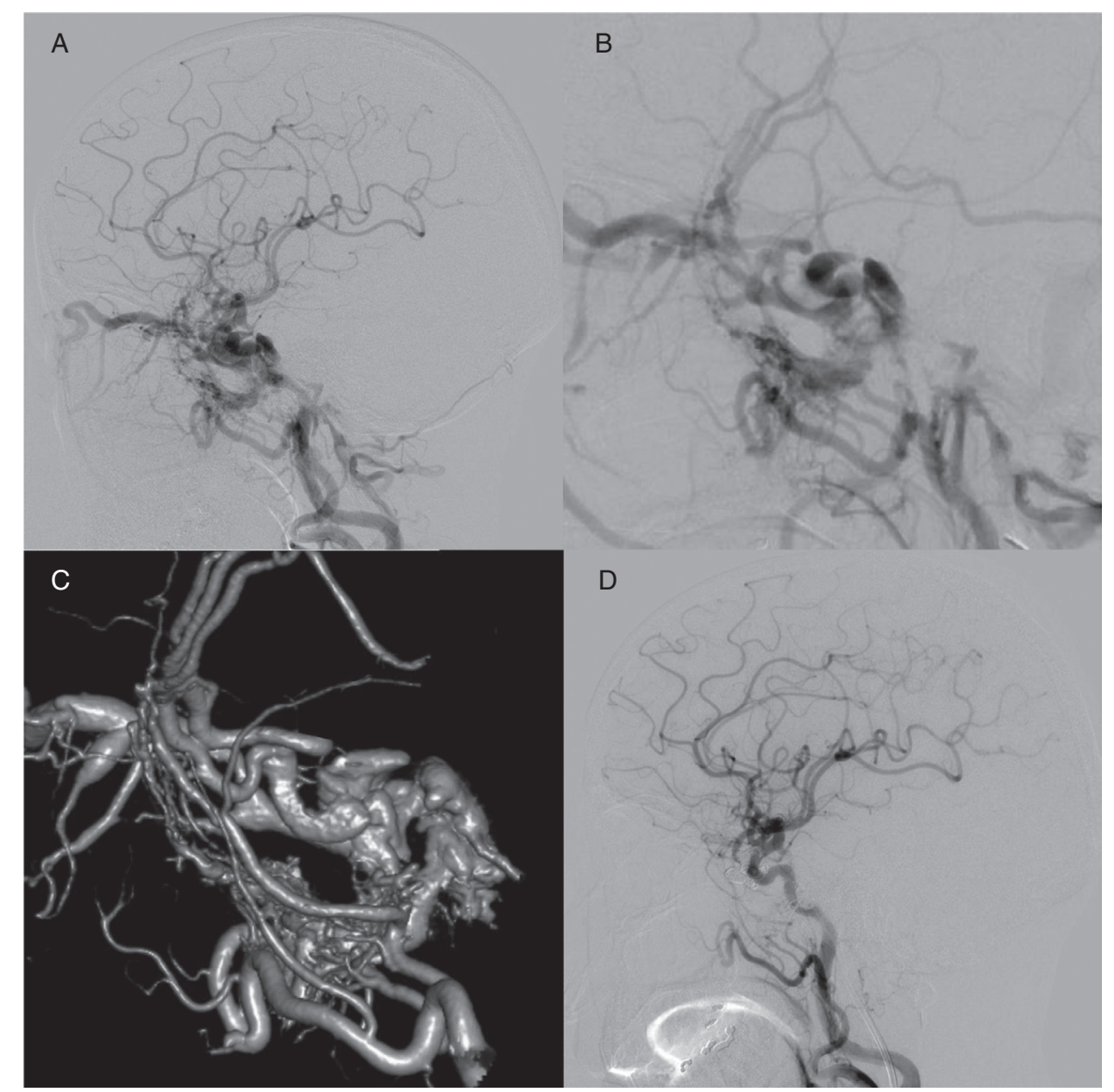

Fig. 4 Angiography in the first treatment. (A) Preoperative angiography of the left common carotid artery. Borden type II CSdAVF was observed. (B and C) Preoperative angiography of the left external carotid artery, $3 \mathrm{D}$ rotography. Shunts from the middle meningeal artery, accessory meningeal artery, artery of foramen rotundum, and ascending pharyngeal artery were present. Blood flowed into the left superior ophthalmic vein, SMCV, IPS, and pterygoid plexus, and it was accompanied by cortical venous reflux. (D) Postoperative angiography of the left common carotid artery. The shunts were lost. CSdAVF: cavernous sinus dural arteriovenous fistula; IPS: inferior petrosal sinus; SMCV: superficial middle cerebral vein

of KTS or KTWS complicated by cerebral aneurysm have been reported and one or more giant cerebral aneurysms were present in three cases of them. ${ }^{4-6)}$ KTS or KTWS was complicated by spinal AVM or fistula in 33 cases $^{7-11)}$ and it tended to develop in the lower thoracic over the lumbar spinal cord. In addition, involvement of the Angiogenic factor with G-patch and FHA domain 1 (AGGF1) and phosphoinositide-3-kinase, catalytic, alpha polypeptide (PIK3CA) genes in KTS has been reported. ${ }^{12,13)}$ Overexpression of the AAGF1 gene promotes expression of flt4, dab2, and EPH receptor B4 (EPHB4), which are venous markers, and it activates protein kinase $\mathrm{B}$ (AKT) playing 


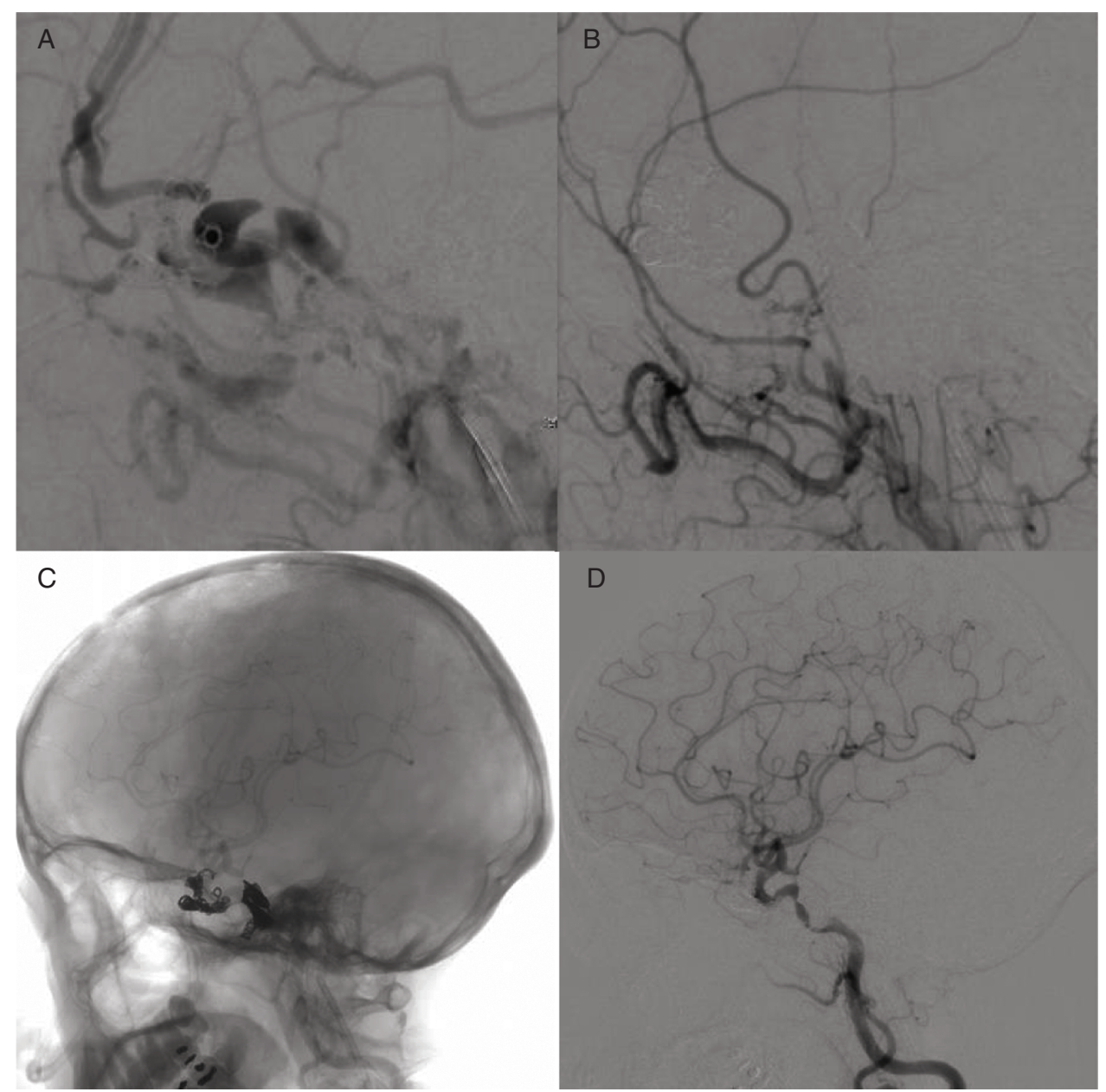

Fig. 5 Angiography in the second treatment. (A) Preoperative angiography of the left external carotid artery. Cortical reflux to the SMCV was noted. (B) Postoperative angiography of the left external carotid artery. (C) Postoperative fluoroscopy. (D) Postoperative angiography of the left common carotid artery. The shunts were lost. SMCV: superficial middle cerebral vein

an important role in cell proliferation, survival, and metabolism, through which venous angiogenesis occurs and leads to vascular malformation. Regarding the PIK3CA gene, when activating mutation occurs in the developmental process, mammalian target of rapamycin having an influence on vascular growth and proliferation is excessively activated and causes angiogenesis and tissue overgrowth. Reduction of vascular lesions and tissue overgrowth in PIK3CA-related overgrowth syndrome by administration of a molecular target drug inhibiting this PIK3CA gene has been reported. ${ }^{14)}$ Sgubin et al. ${ }^{9)}$ reported that KTS may be complicated by spinal AVM due to the influence of angiogenic factor. Angiogenesis due to these gene abnormalities may have contributed to CSdAVF formation in the present patient. On the other hand, Alomari et al. ${ }^{15)}$ denied the association between KTS and spinal AVM. They reviewed cases diagnosed as KTS in previous reports on the association with spinal AVM and found that the cases were actually other similar diseases, such as Congenital lipomatous overgrowth, vascular malformations, and epidermal nevi (CLOVE) syndrome and capillary malformation-AVM (CM-AVM), suggesting difficulty in definitely diagnosing KTS. Gene abnormality causing vascular malformation has been confirmed in several diseases and the association of PWS, which is a KTS-related disease, with RAS p21 protein activator 1 (RASA1) gene abnormality has been reported, ${ }^{16)}$ and involvement of RASA1 and EPHB4 mutations in CM-AVM has also been reported. ${ }^{17)} \mathrm{CM}-\mathrm{AVM}$ was complicated by AVM/fistula in the brain, face, and four limbs in 30\% and when a genetic test was performed in five patients with CM-AVM complicated by spinal AVM/fistula, RASA1 gene abnormality was detected in all five patients in a study. ${ }^{18)}$

To our knowledge, this was the initial case report of KTS with intracranial dAVF. In this patient, the left subclavian 
vein dilated to three times the thickness of the vein on the healthy side accompanying the very large venous malformation in the left upper limb, and phleboliths were also present. Phlebolith is calcified thrombus formed in a vascular lumen with a slow flow velocity and it is considered a characteristic finding of venous malformation. Abnormal coagulation occurs when the volume of the lesion is large and when multiple phleboliths are present. First, we discuss the relationship between dilatation of the left subclavian vein and development of CSdAVF. dAVF development after diagnosing sinus occlusion has been reported, but in our patient, although stenosis of the left IPS was observed at the time of diagnosis, no sinus occlusion inducing dAVF or preceding symptom suggesting its presence was noted. It has been reported that in CSdAVF, the sinus is gradually thrombosed by shunt blood flow, and the posterior outflow path and then anterior outflow path is occluded with progression of the disease stage. ${ }^{19)}$ Applying this to our patient, the disease stage was still early so that the condition was still in the process leading to occlusion of the posterior outflow path. Congestion of the left internal jugular vein was also absent on angiography and no phlebolith suggesting thrombosing was noted. Therefore, it is less likely that dAVF was induced by perfusion injury caused by dilatation of the left subclavian vein. Second, we discuss the relationship between abnormal coagulation and CSdAVF formation in the present patient. The blood test findings before and after treatment are shown in Table 1. A mild decrease in platelets and increases in FDP and D-dimer were noted 1 year before the onset of CSdAVF. FDP and D-dimer markedly increased and fibrinogen decreased immediately after the first TVE and these may have reflected coil-induced thrombosing. The values of the coagulation-fibrinolysis system including TAT and PIC were still abnormal at 3 years after treatment. This abnormal coagulation in this patient was localized intravascular coagulation (LIC) caused by massive consumption of coagulation factor in lesions due to chronic retention of blood in the varix of the affected limb, and this has to be distinguished from Kasabach-Merritt syndrome complicating infantile and pediatric Kaposiform hemangioendothelioma and tufted hemangioma. In addition, local abnormal coagulation may spread to other regions due to sclerotherapy, surgical treatment, fracture, and menstruation and LIC may progress to disseminated intravascular coagulation (DIC). In our patient, thrombosing did not progress in CSdAVF and no finding of DIC was noted. Therefore, it is less likely that abnormal coagulation in pre-existing LIC-induced CSdAVF. The efficacy of perioperative administration of low-molecular-weight heparin to reduce LIC-induced local pain and prevent aggravation to DIC in surgery and sclerotherapy for venous malformation complicated by LIC has been reported. ${ }^{20)}$ Catheter treatment-induced progression of LIC to DIC has not been reported, but it would have been good to consider perioperative administration of low-molecular-weight heparin although there is a problem with coverage of TVE for CSdAVF with national health insurance.

No gene search was performed in this patient and the cause of CSdAVF formation was unclear. Since involvement of gene abnormality inducing angiogenesis in KTS has been reported, its influence on dAVF formation cannot be ruled out. Three years have passed without recurrence after treatment, but continuation of careful follow-up is necessary.

\section{Conclusion}

We performed endovascular treatment in a rare case of CSdAVF complicating KTS and achieved a favorable outcome. Many points are unclear with regard to the cause of dAVF, for which further elucidation of the pathology is expected.

\section{Disclosure Statement}

None of the first and coauthors has conflicts of interest.

\section{References}

1) Al-Shahi R, Bhattacharya JJ, Currie DG, et al: Prospective, population-based detection of intracranial vascular malformations in adults: the Scottish Intracranial Vascular Malformation Study (SIVMS). Stroke 2003; 34: 1163-1169.

2) Kuwayama N, Kubo M, Hori E, et al: Epidemiological survey on dural arteriovenous fistulas in Japan. Grants-in-Aid for Scientific Research, https://kaken.nii.ac.jp/en/grant/ KAKENHI-PROJECT-15591512/.

3) Wassef M, Blei F, Adams D, et al: Vascular anomalies classification: recommendations from the International Society for the Study of Vascular Anomalies. Pediatrics 2015; 136: e203-e214.

4) Star A, Fuller CE, Landas SK: Intracranial aneurysms in Klippel-Trenaunay/weber syndromes: case report. Neurosurgery 2010; 66: E1027-E1028.

5) Kim YW, Kim N, Hwang JM, et al: Teaching neuroImages: multiple giant intracranial aneurysms in Klippel-Trenaunay syndrome. Neurology 2013; 81: e17-e18.

6) Fukaya R, Yanagisawa K, Fukuchi M, et al: Posterior cerebral artery giant aneurysm associated with bilateral internal 
carotid artery occlusion in a Klippel-Trenaunay syndrome patient: a case report. Br J Neurosurg 2017 Oct 26. doi: 10.1080/02688697.2017.1394446. [Epub ahead of print]

7) Rohany $M$, Shaibani A, Arafat $O$, et al: Spinal arteriovenous malformations associated with Klippel-Trenaunay-Weber syndrome: a literature search and report of two cases. AJNR Am J Neuroradiol 2007; 28: 584-589.

8) Misterska E, Glowacki M, Latuszewska J: Female patients' and parents' assessment of deformity- and brace-related stress in the conservative treatment of adolescent idiopathic scoliosis. Spine 2012; 37: 1218-1223.

9) Sgubin D, Kanai R, Di Paola F, et al: Conus medullariscauda arteriovenous malformation and Klippel-Trenaunay syndrome: what is the treatment goal? Neurol Med Chir (Tokyo) 2013; 53: 110-114.

10) Sharma S: Multifocal intradural spinal AVF and renal artery aneurysms in a case of Klippel Trenaunay syndrome (KTS). J Neuroimaging 2010; 20: 386-389.

11) Tokunaga K, Hishikawa $T$, Sugiu K, et al: Combined transarterial and transvenous approach for curative obliteration of Klippel-Trenaunay-weber syndrome-associated spinal perimedullary arteriovenous fistulas. Clin Neuroradiol 2015; 25: 291-294.

12) Tian XL, Kadaba R, You SA, et al: Identification of an angiogenic factor that when mutated causes susceptibility to Klippel-Trenaunay syndrome. Nature 2004; 427 : 640-645.

13) Luks VL, Kamitaki N, Vivero MP, et al: Lymphatic and other vascular malformative/overgrowth disorders are caused by somatic mutations in PIK3CA. J Pediatr 2015; 166: 1048-1054.

14) Venot Q, Blanc T, Rabia SH, et al: Targeted therapy in patients with PIK3CA-related overgrowth syndrome. Nature 2018; 558: 540-546.

15) Alomari AI, Orbach DB, Mulliken JB, et al: KlippelTrenaunay syndrome and spinal arteriovenous malformations: an erroneous association. AJNR Am J Neuroradiol 2010; 31: $1608-1612$.

16) Revencu N, Boon LM, Mulliken JB, et al: Parkes weber syndrome, vein of Galen aneurysmal malformation, and other fast-flow vascular anomalies are caused by RASA1 mutations. Hum Mutat 2008; 29: 959-965.

17) Amyere M, Revencu N, Helaers R, et al: Germline lossof-function mutations in EPHB4 cause a second form of capillary malformation-arteriovenous malformation (CM-AVM2) deregulating RAS-MAPK signaling. Circulation 2017; 136: 1037-1048.

18) Thiex R, Mulliken JB, Revencu N, et al: A novel association between RASA1 mutations and spinal arteriovenous anomalies. AJNR Am J Neuroradiol 2010; 31: 775-779.

19) Satomi J, Satoh K, Matsubara S, et al: Angiographic changes in venous drainage of cavernous sinus dural arteriovenous fistulae after palliative transarterial embolization or observational management: a proposed stage classification. $\mathrm{Neu}$ rosurgery 2005; 56: 494-502.

20) Dompmartin A, Acher A, Thibon P, et al: Association of localized intravascular coagulopathy with venous malformations. Arch Dermatol 2008; 144: 873-877. 